\title{
Solution Structure of the Ubiquitin-conjugating Enzyme UbcH5B
}

\author{
Klaartje Houben ${ }^{1} \dagger$, Cyril Dominguez ${ }^{1} \dagger$, Frederik M. A. van Schaik ${ }^{2}$ \\ H. Th. Marc Timmers ${ }^{2}$, Alexandre M. J. J. Bonvin ${ }^{1}$ and Rolf Boelens ${ }^{1 *}$
}

${ }^{1}$ Department of NMR

Spectroscopy, Bijvoet Center for Biomolecular Research, Utrecht University, Padualaan 8, 3584 $\mathrm{CH}$ Utrecht, The Netherlands

${ }^{2}$ Department of Physiological Chemistry, University Medical Center Utrecht

Universiteitsweg 100, 3584 CG Utrecht, The Netherlands
The ubiquitination pathway is the main pathway for protein degradation in eukaryotic cells. The attachment of ubiquitin to a substrate protein is catalyzed by three types of enzymes, namely a ubiquitin activating enzyme (E1), a ubiquitin-conjugating enzyme (E2), and a ubiquitin ligase (E3). Here, the structure of the human ubiquitin-conjugating enzyme (E2) $\mathrm{UbcH} 5 \mathrm{~B}$ has been solved by a combination of homology modeling, NMR relaxation data and automated NOE assignments. Comparison to E2 structures solved previously by X-ray crystallography or NMR shows in all cases the same compact fold, but differences are observed in the orientation of both $\mathrm{N}$ and $\mathrm{C}$-terminal $\alpha$-helices. The $\mathrm{N}$-terminal helix that is involved in binding to ubiquitin ligases (E3) displays a different position, which could have consequences for precise E2-E3 recognition. In addition, multiple conformations of the side-chain of Asn77 are found in solution, which contrasts the single hydrogen-bonded conformation in the crystal structures of E2 enzymes. The possible implication of this conformational freedom of Asn77 for its catalytic function is discussed.

(C) 2004 Elsevier Ltd. All rights reserved.

Keywords: ubiquitination; NMR; automated NOE assignment; backbone dynamics; diffusion anisotropy regulatory functions such as in signal transduction, transcription regulation, chromatin remodeling and DNA repair. ${ }^{8}$ In all cases the attachment of ubiquitin moieties to the substrate is catalyzed by three enzymes. First, an E1, or ubiquitin-activating enzyme, forms a thiol ester with the carboxylterminal group of ubiquitin. Second, the ubiquitin is transferred to the ubiquitin-conjugating enzyme (E2). Finally, a ubiquitin ligase (E3) transfers ubiquitin from E2 to the substrate protein. In human cells, one E1, about $30 \mathrm{E} 2 \mathrm{~s}$ and at least 400 putative E3s have been identified. The selectivity and specificity of ubiquitination depends on the E2-E3 and E3-target complexes. Comparison of the different E2 and E3 structures and their complexes should shed light on the specificity encountered in such complex formation. Therefore structures of various E2s and E3s are needed. To date, ten structures of E2 enzymes from different species have been solved in their free form, nine by X-ray crystallography $^{9-18}$ and one by NMR. ${ }^{19}$ Furthermore, five structures of three different E2s in complex with various other proteins have been solved by X-ray crystallography. ${ }^{16,20-23}$ All E2 structures possess the same compact fold corresponding to an $\mathrm{N}$-terminal $\alpha$-helix, followed by a 
four-stranded antiparallel $\beta$-sheet and three C-terminal $\alpha$-helices. Moreover the long stretch that contains the active site cysteine residue is very welldefined in all structures. The cysteine residue is surrounded by loops that form a slight depression on the protein surface, and is solvent-exposed in all known E2 structures. It has been postulated that the mechanism of transfer of ubiquitin from E1 to E2 and from E2 to the substrate resembles that of a thiol protease. ${ }^{2}$ Based on the crystal structure of Ubc9, ${ }^{11}$ some conserved residues around the active site were proposed as possible catalysts of isopeptide bond formation. Among these residues was one highly conserved asparagine residue (Asn77 in $\mathrm{UbcH5B}$ ). The importance of this asparagine residue was confirmed by mutagenesis experiments of three other E2s that revealed its necessity for efficient isopeptide bond formation in E2-catalyzed ubiquitin conjugation. ${ }^{24}$ In the different crystal structures of Ubcs, however, this asparagine is hydrogen-bonded to the loop connecting helix $\mathrm{H} 2$ and $\mathrm{H} 3$ and pointing away from the $\mathrm{E} 2$ cysteine and therefore cannot participate in the enzymatic reaction. This implies that a repositioning of this asparagine residue must occur to catalyze the isopeptide bond formation. ${ }^{11,24}$

Here we report the structure of the ubiquitinconjugating enzyme UbcH5B determined by NMR spectroscopy using a combination of homology modeling, diffusion anisotropy restraints and automated nuclear Overhauser effect (NOE) assignment. Dynamical properties of $\mathrm{UbcH} 5 \mathrm{~B}$ were assessed from ${ }^{15} \mathrm{~N}$ relaxation measurements, which show limited motion for the major part of the protein backbone. The relaxation data $\left(R_{2} / R_{1}\right)$ have been translated into diffusion anisotropy restraints and used in the structure calculations. ${ }^{25-27}$ The final structure, which is well defined, possesses the canonical E2 fold, but differences in the position of the $\mathrm{N}$ and $\mathrm{C}$-terminal helices with respect to the core of the protein are observed. Since the N-terminal helix is involved in binding to ubiquitin ligases, the position of this helix in the different structures may be important for differentiating between various E3 ligases. Moreover, our NMR data suggest that the important asparagine residue (Asn77) undergoes conformational exchange in solution and does not adopt a single
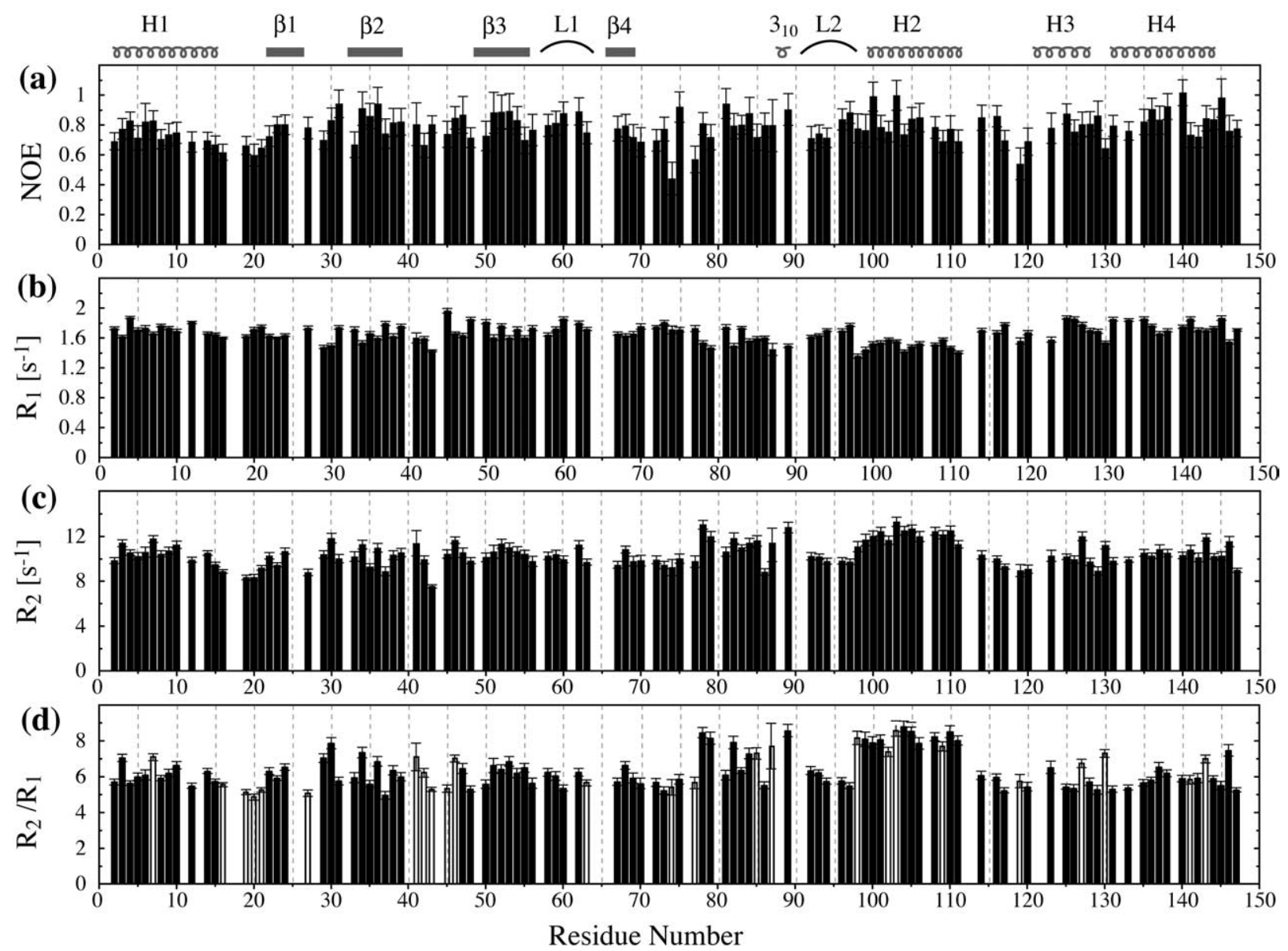

Figure $1 .{ }^{15} \mathrm{~N}$ relaxation parameters of $\mathrm{UbcH} 5 \mathrm{~B}$ at $300 \mathrm{~K}$ and $500 \mathrm{MHz}$. (a) Heteronuclear NOE values, (b) $R_{1}$ relaxation rates, (c) $R_{2}$ relaxation rates from CPMG experiments and (d) $R_{2} / R_{1}$ ratios. The filled bars in (d) represent the 87 selected ratios. The secondary structure elements and the two loops L1 and L2 involved in the interaction with the CNOT4 E3 ligase $^{32}$ are indicated on top. 

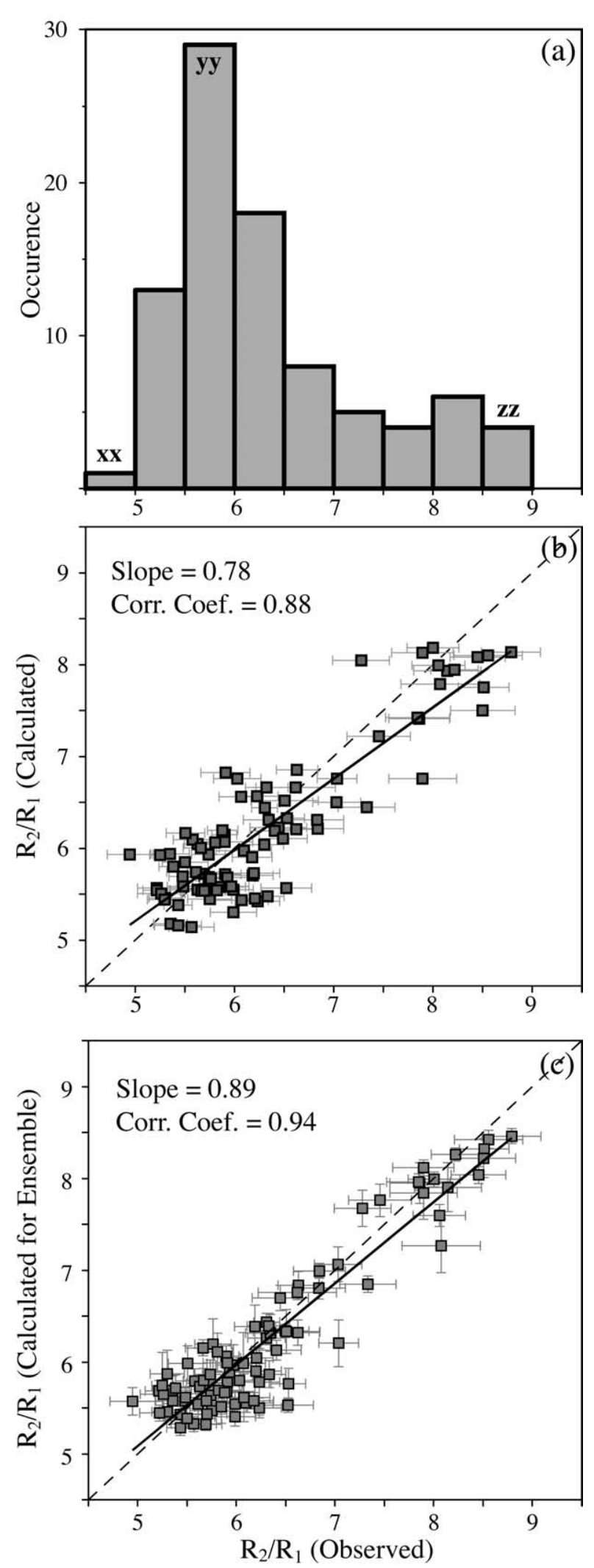

Figure 2. Diffusion anisotropy of UbcH5B. (a) Histogram of $R_{2} / R_{1}$ ratios for $\mathrm{UbcH} 5 \mathrm{~B}$. The shape of the histogram indicates that the diffusion tensor of $\mathrm{UbcH} 5 \mathrm{~B}$ has a rod shape with a small rhombic component. Observed versus back-calculated $R_{2} / R_{1}$ ratios (b) for the homology model of UbcH5B and (c) for the ensemble of ten lowest energy structures of UbcH5B. The error bars correspond to the experimental errors (horizontal) and to well-defined position of its side-chain. This conformational rearrangement may have a critical role in catalysis.

\section{Results and Discussion}

\section{Assignment of UbcH5B, secondary structure, and hydrogen bond prediction}

The assignment of $\mathrm{UbcH} 5 \mathrm{~B}$ has been reported. ${ }^{28}$ However, the data were collected at different temperature, $\mathrm{pH}$, and salt concentration than used here. Therefore, for the backbone assignment of $\mathrm{UbcH} 5 \mathrm{~B}$ in our conditions $(300 \mathrm{~K}, \mathrm{pH} 7.0,150 \mathrm{mM}$ $\mathrm{KCl})$, 2D $\left({ }^{15} \mathrm{~N}-{ }^{1} \mathrm{H}\right)-\mathrm{HSQC}, 2 \mathrm{D}\left({ }^{13} \mathrm{C}-{ }^{1} \mathrm{H}\right)-\mathrm{HSQC}, 3 \mathrm{D}$ $\mathrm{HNCO}$, 3D HNCACB, and 3D CBCA(CO)NH were recorded. The side-chain assignment was performed using 3D TOCSY- $\left({ }^{15} \mathrm{~N}-{ }^{1} \mathrm{H}\right)-\mathrm{HSQC}, 3 \mathrm{D}$ $\mathrm{H}(\mathrm{C}) \mathrm{CH}-\mathrm{TOCSY}$ and 3D (H)CCH-TOCSY spectra (for a review see Sattler et al. ${ }^{29}$ ). All residues except the 16 proline residues were assigned. Finally, 85\% of all observable protons could be assigned. All E2 structures previously solved contain a proline (Pro61 in UbcH5B) in a cis conformation. Analysis of our NOE spectra could not discriminate whether this proline was in a cis or trans conformation in UbcH5B. Moreover, in the 3D (H)CCH-TOCSY spectrum nine proline spin-systems could be identified, but none of the $C^{\beta}$ and $C^{\gamma}$ resonances had chemical shifts typical for a cis conformation. Therefore both cis and trans conformations were allowed during the structure calculation (see below). Dihedral angles of $\mathrm{UbcH} 5 \mathrm{~B}$ were predicted with the program TALOS ${ }^{30}$ based on the $C^{\alpha}$ and $C^{\beta}$ chemical shifts. These predictions correlate well with the consensus secondary structure elements found in known E2 structures (data not shown). Amide protons involved in a hydrogen bond were identified from proton-deuterium exchange as described in Materials and Methods and 27 hydrogen bond restraints were used in the structure calculation, where the hydrogen bond acceptor was defined ambiguously. In agreement with the various E2 structures, these 27 amide protons are located mainly in the second, third and fourth $\beta$-strands as well as in helix H2. Amide protons located in the $\mathrm{N}$ and $\mathrm{C}$-terminal $\boldsymbol{\alpha}$-helices, as well as in the first $\beta$-strand were poorly or not protected. Consistently, amide protons of the second $\beta$-strand that are hydrogen bonded to the first $\beta$-strand in the known E2 structures were also not protected. Interestingly, few amide protons that are present in loops were also protected. These are Tyr45 in the loop that connects the second and third $\beta$-strands,

the standard deviations of the back-calculated values over the ensemble (vertical). The thick lines represent the linear best fit through the data points, with a slope of 0.78 and 0.89 and a correlation coefficient of 0.88 and 0.94 , respectively. 
Lys63 in the L1 loop important for the E3 recognition, and Ile78 close to the active site cysteine.

\section{Diffusion anisotropy and refinement of the homology model}

The amide ${ }^{15} \mathrm{~N}$ relaxation rates $R_{1}, R_{2}$ and $\left\{{ }^{1} \mathrm{H}\right\}$ NOE for 112 out of the 131 non-proline residues of UbcH5B are shown in Figure 1. Residues that have overlapping peaks in the HSQC spectrum were discarded in the analysis, as well as Glu122, which has only a very low intensity peak. UbcH5B has a rather rigid backbone, with an average heteronuclear NOE value of $0.78( \pm 0.09)$ for all residues and $0.80( \pm 0.08)$ for residues located in secondary structure elements. The average $R_{1}$ and $R_{2}$ values are $1.67( \pm 0.13) \mathrm{s}^{-1}$ and $10.5( \pm 1.1) \mathrm{s}^{-1}$, respectively, for the entire backbone and $1.68( \pm 0.12) \mathrm{s}^{-1}$ and $10.6( \pm 0.9) \mathrm{s}^{-1}$, respectively, for the secondary structure elements. The $R_{2} / R_{1}$ ratios, which are illustrative of the rotational diffusion characteristics of a protein, are presented in Figure 1(d). The filled bars represent the $87 R_{2} / R_{1}$ ratios that were selected for the determination of the rotational diffusion parameters of the protein. From the average $R_{2} / R_{1}$ ratio $(6.3 \pm 0.9)$ the isotropic diffusion tensor constant was estimated to be $1.9( \pm 0.3) \times 10^{7} \mathrm{~s}^{-1}$, which gives an apparent correlation time of $8.6( \pm 1.2) \mathrm{ns}$. The histogram of $R_{2} / R_{1}$ ratios in Figure 2(a), however, immediately reveals that $\mathrm{UbcH} 5 \mathrm{~B}$ does not tumble isotropically in solution. High $R_{2} / R_{1}$ ratios are found for the residues located in helix $\mathrm{H} 2$, caused by both their high $R_{2}$ rates $\left(>11.6 \mathrm{~s}^{-1}\right)$ and low $R_{1}$ rates $\left(<1.54 \mathrm{~s}^{-1}\right)$. The possibility that the high $R_{2}$ rates in this helix are caused by conformational exchange could be excluded, since no differences were observed between the ${ }^{15} \mathrm{~N} R_{2}$ rates determined from both CPMG and $T_{1 \rho}$ experiments (data not shown). In addition, relaxation dispersion profiles measured at both $500 \mathrm{MHz}$ and $700 \mathrm{MHz}$, that are very sensitive to conformational exchange, did not give any indication for such effects in helix H2 (data not shown). In a previous study of the interaction of $\mathrm{UbcH} 5 \mathrm{~B}$ with the RING domain of CNOT4 we generated a homology model of UbcH5B based on the structure of the highly homologous yeast Ubc4 (Figure $3(\mathrm{a}))^{32}$ In this model, helix H2 is approximately parallel to the long axis of the protein, which would explain the high $R_{2} / R_{1}$ ratios in this part of the protein. However, as shown in Figure 2(b), the backcalculated $R_{2} / R_{1}$ values still deviate from the experimental values with a reduced $\chi^{2}$ of 4.59 (for details, see Materials and Methods). The deviation can be mainly attributed to the orientation of helix $\mathrm{H} 2$. The homology model was therefore refined in explicit water, using TALOS-derived dihedral angles and diffusion anisotropy restraints. Six independent structure refinements (three with Pro61 in a cis and three with Pro61 in a trans conformation) were performed including the $R_{2} / R_{1}$ orientational restraints, starting from three different estimates of the anisotropy $A$ and rhombicity $\eta$ of the diffusion tensor: (i) 1.61 and 0.33 , (ii) 1.68 and 0.27 and (iii) 1.64 and 0.31 , which were obtained as described in Materials and Methods. The average reduced $\chi^{2}$ value decreased from 4.59 to $2.26( \pm 0.23), 2.15( \pm 0.21)$ and $2.03( \pm 0.20)$, respectively, using optimized values of $A$ and $\eta$ for the three refined sets that each consisted of 20 models. For the 60 refined models, the optimized anisotropy $A$ was 1.70 and the optimized rhombicity $\eta 0.30$. These were used as starting values for the diffusion tensor in all subsequent structure calculations. The average pairwise backbone RMSD between the 60 refined models was $0.47( \pm 0.04) \AA$ and the backbone RMSD from the original homology model was $0.79( \pm 0.04) \AA$.

\section{Model-based automated NOE assignment and structure calculations of UbcH5B}

In order to facilitate and speed up structure determination by NMR, approaches have been developed that combine structure calculations and automated assignment of NOE spectra in an iterative manner. ${ }^{33-35}$ For large proteins, however, the ambiguity in the assignment increases rapidly due to peak overlap in the spectra and makes automated assignment in that case difficult, causing poor convergence of the calculated structures to a unique fold. It has been suggested that the use of starting models can assist and speed up automated NOE assignment procedures. ${ }^{36-38}$

The automated NOE assignment and structure calculations of $\mathrm{UbcH} 5 \mathrm{~B}$ were performed with a version of ARIA1.2 ${ }^{33}$ that we modified to include diffusion anisotropy restraints (see Materials and Methods). This modified version of ARIA has been made available via the ARIA homepage. $\dagger$ A total of 7493 NOEs were obtained from two 2D NOE spectra recorded in $\mathrm{H}_{2} \mathrm{O}$ and in ${ }^{2} \mathrm{H}_{2} \mathrm{O}$ on a $900 \mathrm{MHz}$ spectrometer and two $3 \mathrm{D}\left({ }^{15} \mathrm{~N}-{ }^{1} \mathrm{H}\right)-$ NOESY-HSQC spectra recorded at $750 \mathrm{MHz}$ and $900 \mathrm{MHz}$. From the two 3D $\left({ }^{15} \mathrm{~N}-{ }^{1} \mathrm{H}\right)-\mathrm{NOESY}-$ HSQC and the two 2D NOE spectra 1354, 1002, 4784 (on both sides of the diagonal), and 353 (aromatic and $\alpha$ protons region) NOE peaks were extracted, respectively. 404 intra and 284 sequential NOEs were manually assigned in the 2D NOE spectrum in $\mathrm{H}_{2} \mathrm{O}, 69$ intra and 24 sequential NOEs in the $2 \mathrm{D}$ NOE spectrum in ${ }^{2} \mathrm{H}_{2} \mathrm{O}$, and 795 intra and 612 sequential in the two 3D spectra. The chemical shifts of the corresponding nuclei were adjusted based on these assignments, which allowed the use of tight chemical shift tolerances in the automated NOE assignment protocol: $0.02 \mathrm{ppm}$ for the ${ }^{1} \mathrm{H}$ chemical shift values and $0.2 \mathrm{ppm}$ in the ${ }^{15} \mathrm{~N}$ dimension. In addition, 77 dihedral angle restraints (both $\phi$ and $\psi$ derived from TALOS ${ }^{30}$ ), 27 hydrogen bond restraints derived from proton-deuterium exchange (see Materials and Methods) and 87

$\dagger$ http://www.pasteur.fr/recherche/unites/Binfs/aria 

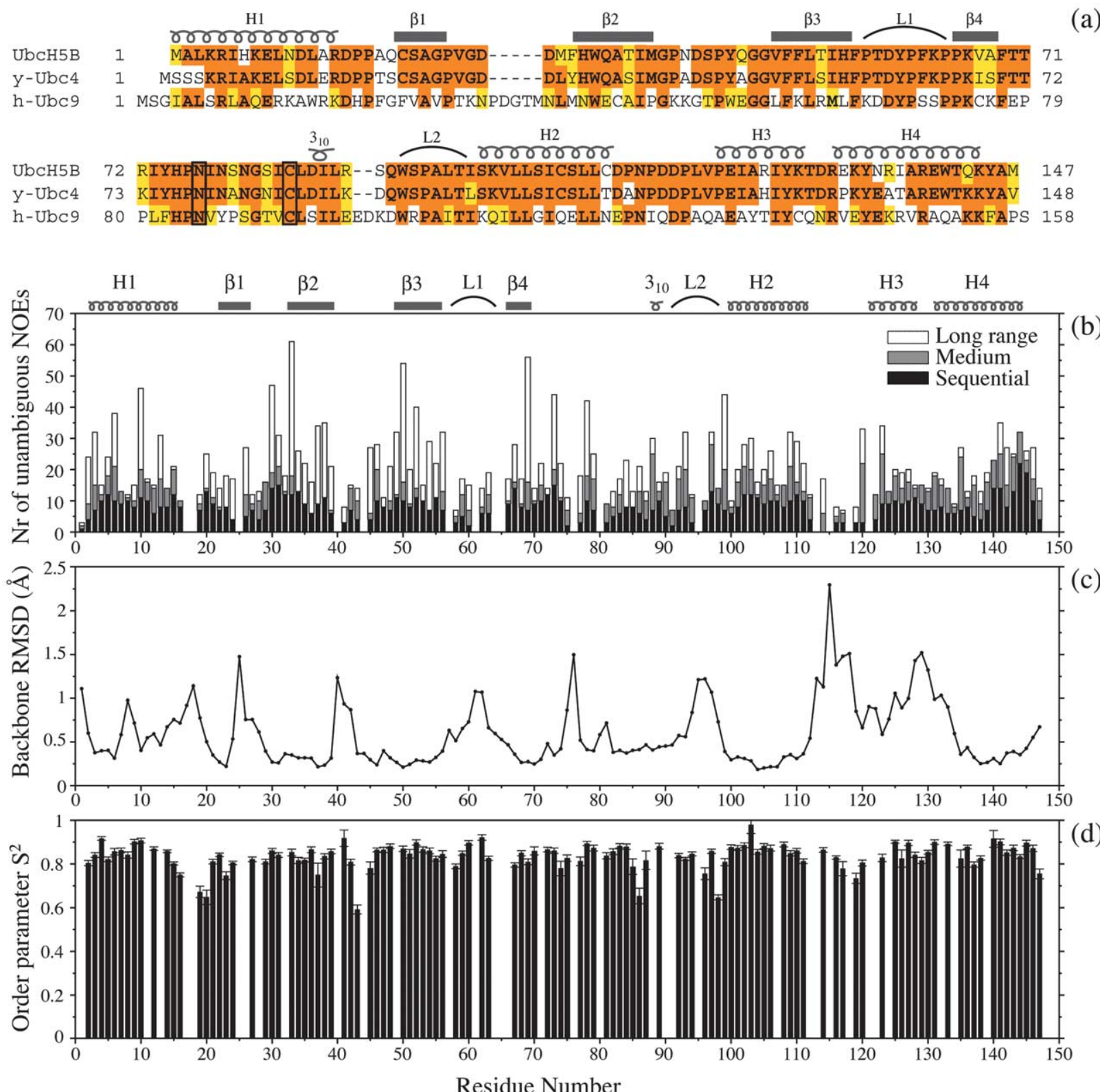

(d)

Figure 3. Structural and dynamical data of the UbcH5B NMR structure. (a) Sequence alignment of UbcH5B, yeast Ubc4 and human/murine Ubc9. Identical residues are colored orange and homologous residues yellow. The active site cysteine 85 and asparagine 77 are indicated by the black boxes. Secondary structure elements as found for UbcH5B are illustrated. (b) Distribution of the number of unambiguous NOE restraints over the protein sequence. The numbers indicated are the final numbers used in the structure calculation. Secondary structure elements and loops L1 and L2 are indicated. (c) Average backbone RMSD for each residue from the mean. The values shown are the average RMSD calculated from the ensemble of ten structures. (d) Generalized order parameter $S^{2}$ obtained from the model-free analysis of the ${ }^{15} \mathrm{~N}$ relaxation data.

diffusion anisotropy restraints were used in the structure calculation.

Three successive ARIA runs (runs 1-3) were performed, each consisting of nine iterations and a final refinement in explicit water for the last run. In run 1, the 60 refined models (30 with Pro61 in a cis conformation and 30 in a trans conformation) were used to perform the automated assignment of the NOEs. No structures were calculated at this level yet. The distance violation tolerance was set to $1.0 \AA$ with an ambiguity cut-off of 0.90 resulting in the assignment of 1973 unambiguous and 1790 ambiguous NOEs after merging of the four spectra. Based on these assignments two subsequent runs of structure calculations were performed including TALOS-derived dihedral angles and $R_{2} / R_{1}$-derived diffusion anisotropy restraints. Run 2 started from an extended structure, using the NOE restraints from run 1 . The topology file was modified to make sure that Pro61 could adopt either a cis or a trans conformation. Run 2 consisted of nine ARIA iterations, in which the structures gradually 
converged and in which the ambiguous NOE restraints could be reassigned, recalibrated and rejected if violated in more than $50 \%$ of the 20 best structures of the previous iteration. For the last two iterations the anisotropy $A$ and rhombicity $\eta$ of the diffusion tensor were optimized using a grid search, giving a value of 1.72 for $A$ and a value of 0.28 for $\eta$. This run resulted in 2172 unique unambiguous and 1307 ambiguous NOE assignments and in an already well-defined ensemble of structures (average backbone RMSD from the mean structure of $0.60( \pm 0.14) \AA$ for the secondary structure elements). In the final run, run 3 , the unambiguous and ambiguous NOE restraints from run 2 were fixed. In addition the NOE peaks (in total 2574) that were not previously converted into either unambiguous or ambiguous restraints were added into the calculation. The initial automated NOE assignment for these peaks was based on the ten final structures of run 2 (eight structures contained Pro61 in a cis conformation). The optimized values of $A$ and $\eta$ from run 2 were used for the diffusion anisotropy restraints. In addition, the hydrogen bond restraints were added in run 3. After each iteration the diffusion tensor was optimized, giving a final value of 1.72 and 0.23 for $A$ and $\eta$, respectively. This run finally resulted in the assignment of 2982 unambiguous (2299 unique unambiguous NOEs) and 1783 ambiguous NOEs (Table 1) derived from 3769 assigned cross-peaks $(79 \%)$ in the NOESY in $\mathrm{H}_{2} \mathrm{O}, 240(70 \%)$ in the NOESY in ${ }^{2} \mathrm{H}_{2} \mathrm{O}, 1250$ (92\%) in the 3D-NOESYHSQC spectrum recorded at $750 \mathrm{MHz}$ and 883 $(88 \%)$ in the 3D-NOESY-HSQC spectrum recorded at $900 \mathrm{MHz}$. Figure 3(b) shows the distribution of the unambiguous NOEs over the polypeptide sequence. In general a large number of NOEs are found in the secondary structure elements.

After water refinement, 15 out of the best 20 structures contained Pro61 in a cis conformation. Structural analysis was therefore performed on the ten best structures that contain Pro61 in a cis conformation. The structures satisfy the experimental restraints, with no distance violations larger than $0.45 \AA$, and are well-defined, with an average backbone RMSD from the mean for the secondary structure elements of the ten lowest energy structures of $0.35( \pm 0.04) \AA$ (all heavy atoms: $0.71( \pm 0.09) \AA$ ) (Table 1$)$. Figure 3(c) clearly shows that the RMSD values in the loops between the secondary structure elements are significantly higher. This correlates with the lower number of NOEs found in those regions. The structural statistics are presented in Table 1 . The average backbone RMSD between the ensemble of structures and the original homology model is $2.24( \pm 0.05) \AA$, whereas for the secondary structures the backbone RMSD is $2.21( \pm 0.09) \AA$. The differences between the structure of $\mathrm{UbcH} 5 \mathrm{~B}$ and the homology model are most prominent in the orientation of the first and last two $\alpha$-helices. The average reduced $\chi^{2}$ for the diffusion anisotropy restraints is $3.39( \pm 0.32)$ for the ensemble and 3.06
Table 1. Structural statistics of the ensemble of UbcH5B structures

\begin{tabular}{|c|c|}
\hline \multicolumn{2}{|l|}{ Number of experimental restraints } \\
\hline Intra-residue unambiguous NOEs & 900 \\
\hline Sequential unambiguous NOEs & 530 \\
\hline Medium-range unambiguous NOEs & 345 \\
\hline Long-range unambiguous NOEs & 524 \\
\hline Total unambiguous NOEs & 2299 \\
\hline Total ambiguous NOEs & 1783 \\
\hline Dihedral angles ${ }^{\mathrm{a}}$ & $(77 \phi+77 \psi) 154$ \\
\hline Hydrogen bonds ${ }^{\mathrm{b}}$ & 27 \\
\hline Diffusion anisotropy $^{\mathrm{c}}$ & 87 \\
\hline \multicolumn{2}{|l|}{ RMSD $(\AA)$ from the mean } \\
\hline All backbone atoms & $0.69 \pm 0.09$ \\
\hline All heavy atoms & $1.15 \pm 0.13$ \\
\hline Secondary structure backbone atoms ${ }^{\mathrm{d}}$ & $0.35 \pm 0.04$ \\
\hline Secondary structure heavy atoms ${ }^{\mathrm{d}}$ & $0.72 \pm 0.09$ \\
\hline \multicolumn{2}{|c|}{ Non-bonded energy values ${ }^{\mathrm{e}}$ after water-refinement $\left(\mathrm{kcal}_{\mathrm{mol}}{ }^{-1}\right)$} \\
\hline E vd Waals & $-1485 \pm 22$ \\
\hline$E$ electrostatic & $-5187 \pm 91$ \\
\hline \multicolumn{2}{|l|}{ RMSD from idealized covalent geometry } \\
\hline Bonds $(\AA)$ & $0.0062 \pm 0.002$ \\
\hline Angles $\left({ }^{\circ}\right)$ & $0.75 \pm 0.24$ \\
\hline Impropers $\left({ }^{\circ}\right)$ & $2.36 \pm 0.75$ \\
\hline \multicolumn{2}{|l|}{ RMSD from experimental data } \\
\hline Distance $(\AA)$ & $0.06 \pm 0.01$ \\
\hline Dihedral $\left({ }^{\circ}\right)$ & $2.02 \pm 0.35$ \\
\hline Diffusion anisotropy & $0.38 \pm 0.01$ \\
\hline \multicolumn{2}{|c|}{ Restraint violations in more than $50 \%$ of the structures ${ }^{\mathrm{f}}$} \\
\hline Distance $(>0.3 \AA)$ & 4 \\
\hline Dihedral $\left(>5^{\circ}\right)$ & 5 \\
\hline Diffusion anisotropy $(>0.7)$ & 5 \\
\hline Hydrogen bonds & 0 \\
\hline \multicolumn{2}{|l|}{ Ramachandran analysis } \\
\hline Residues in most favored regions (\%) & $72.3 \pm 2.5$ \\
\hline Residues in additional allowed regions (\%) & $22.6 \pm 2.4$ \\
\hline Residues in generously allowed regions (\%) & $4.1 \pm 1.3$ \\
\hline Residues in disallowed regions (\%) & $1.0 \pm 0.8$ \\
\hline \multicolumn{2}{|c|}{$\begin{array}{l}\mathbf{a} \text { The dihedral angles are derived from } \text { TALOS }^{30} \text { prediction } \\
\text { based on } C^{\alpha} \text { and } C^{\beta} \text { chemical shifts. } \\
\text { b The hydrogen bond restraints are derived from hydrogen- } \\
\text { deuterium exchange experiments. } \\
\text { c The diffusion anisotropy restraints are derived from the } R_{2} / \\
R_{1} \text { ratios. } \\
\text { d Secondary structure elements comprise residues } 2-15,22-24 \text {, } \\
32-38,49-55,66-69,99-111,122-128 \text {, and } 131-144 \text {. } \\
\text { e The non-bonded energies were calculated with the OPLS } \\
\text { parameters using a } 8.5 \AA \text { cutoff. } \\
\text { f No NOE distance restraint was violated by more than } 0.45 \AA \text {, } \\
\text { no dihedral angles by more than } 10.4^{\circ} \text { and no diffusion } \\
\text { anisotropy by more than } 0.99 \text {. }\end{array}$} \\
\hline
\end{tabular}

for the representative structure. Experimental against back-calculated $R_{2} / R_{1}$ ratios for the ensemble are plotted in Figure 2(c). The correlation coefficient of 0.94 indicates a good agreement between the calculated and experimental values. The large number of NOEs results in a reduced $\chi^{2}$ that is slightly higher than for the refined homology models (see above). The latter would, however, violate more than $1000 \mathrm{NOE}$ restraints.

\section{Solution structure of UbcH5B}

The structure of UbcH5B is composed of a fourstranded antiparallel $\beta$-sheet and four $\alpha$-helices (Figure 4). The N-terminal $\alpha$-helix (residues $2-15$ ) is followed by the four-stranded $\beta$-sheet (residues 22$24,32-37,49-55,66-69)$. The first $\beta$-strand, however, 

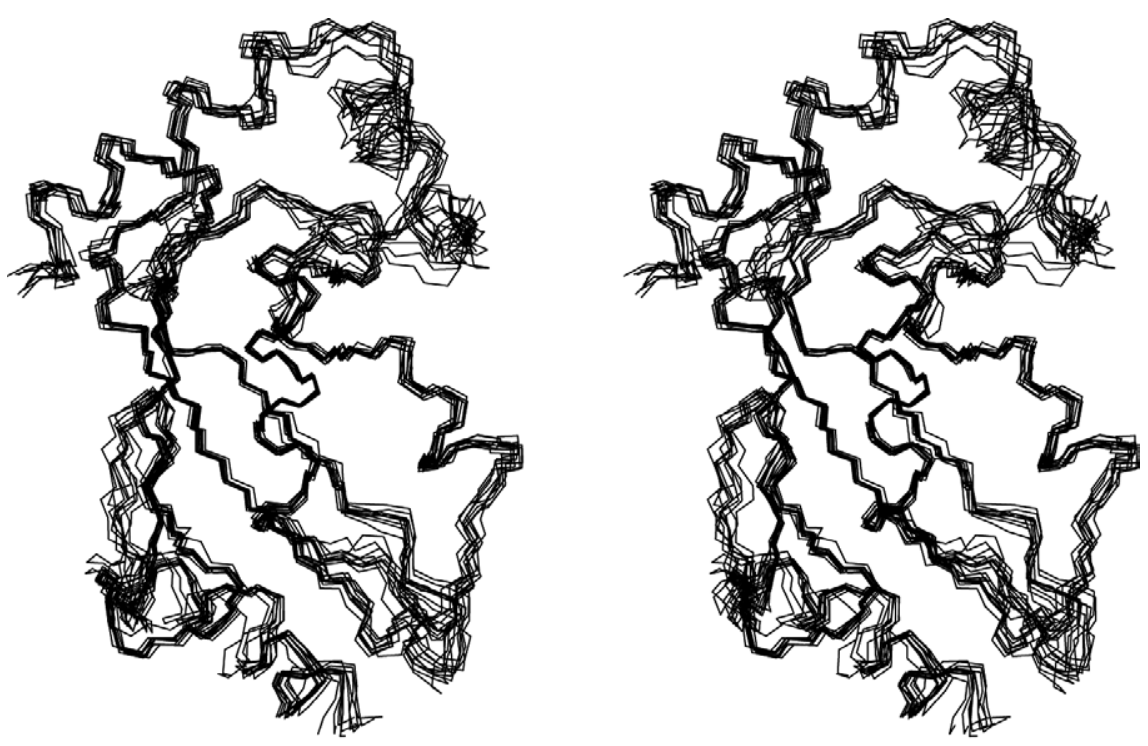

Figure 4. NMR structure of UbcH5B. Stereoview of the ten best structures. This Figure was generated using the program MOLMOL. ${ }^{65}$

is only present in two of the ten analyzed structures. This is consistent with the deuterium exchange experiments where amide protons at the interface between strands $\beta 1$ and $\beta 2$ were not protected. A long extended stretch (residues 71-86), a short $3_{10}$ helix (residues 87-90) and a loop (residues 90-98) connect the last $\beta$-strand to the second $\alpha$-helix (residues 99-111). The second $\alpha$-helix (H2) is parallel to the long axis of the protein in agreement with the diffusion anisotropy data. The C-terminal part of the protein is composed of two $\alpha$-helices (residues 122-128 and 131-145). The core of the protein, which consists of the $\beta$-sheet, the long extended stretch that contains the active site cysteine and the $\alpha$-helix H2, is very well defined with an average backbone RMSD from the mean of $0.40( \pm 0.07) \AA$ (all heavy atoms, $1.07( \pm 0.16) \AA$ ). The two $\mathrm{C}$-terminal $\alpha$-helices are slightly more disordered, probably due to a low number of longrange NOEs in this region.

\section{Backbone dynamics of UbcH5B}

The average anisotropic rotational diffusion tensor for the ensemble of ten UbcH5B structures was analyzed with the program TENSOR2. ${ }^{39}$ This resulted in an overall rotational correlation time $\tau_{\mathrm{c}}$ of $8.63( \pm 0.02) \mathrm{ns}$, an anisotropy $A$ of $1.70( \pm 0.03)$ and a rhombicity $\eta$ of $0.17( \pm 0.07)$. The overall rotational correlation time corresponds nicely with the correlation time expected for a monomeric protein of this size at $300 \mathrm{~K}$ and with the value of $8.5 \mathrm{~ns}$ calculated by the program HYDRONMR. ${ }^{40,41}$ Using the determined anisotropic diffusion tensor, the ${ }^{15} \mathrm{~N}$ relaxation rates (Figure 1 ) were analyzed in TENSOR2 using the model-free ${ }^{42}$ approach based on the representative structure. The resulting order parameters $S^{2}$ are shown in Figure 3(d) and colorcoded on the structure in Figure 5 . The average order parameter is $0.83( \pm 0.06)$. The most flexible parts in $\mathrm{UbcH} 5 \mathrm{~B}$ are the loop connecting the $\mathrm{N}$-terminal helix $\mathrm{H} 1$ with the $\beta$-sheet and the loop connecting the strand $\beta 2$ with $\beta 3$. Some residues with lower order parameters are also present in the long extended stretch that contains the active site cysteine. In loop L2, which is known to be involved in binding to E3 ligases, ${ }^{21}$ Thr98 has a lower order parameter. Furthermore, flexible residues are found in the loop between helix H2 and H3. This loop forms a "lid" on top of the active site Cys85 with in particular the side-chain of Leu119 in close proximity to the cysteine side-chain. The flexibility in this loop could therefore affect both ubiquitin binding and ubiquitination of the substrate.

\section{Comparison of UbcH5B with other Ubc enzymes}

For comparing the structure and dynamics of $\mathrm{UbcH} 5 \mathrm{~B}$ with other Ubc enzymes, we focus on yeast Ubc4, ${ }^{10}$ which is highly homologous to $\mathrm{UbcH} 5 \mathrm{~B}$ and on human/murine Ubc $9^{11,98}$ of which the backbone dynamics have been studied by NMR before (for sequence alignment see Figure 3(a)). ${ }^{43}$ The core domain of UbcH5B is very similar to the X-ray structure of Ubc4 (backbone RMSD of $1.12 \AA$ ) (Figure 6). This close structural resemblance of the core domain is a common feature of all $\mathrm{Ubc}$ structures, which all have an RMSD within $1.3 \AA$ for this part of the structure. It is interesting to note that the $3_{10}$ helix (residues 87-89) is conserved among all E2s and that the long extended stretch that contains the active site cysteine (residues 82 to 89 in $\mathrm{UbcH}$ 5B) has a very similar conformation in all Ubc structures, in particular at the active site cysteine. On the basis of the ${ }^{15} \mathrm{~N}$ relaxation experiments, clear differences in dynamics between $\mathrm{UbcH} 5 \mathrm{~B}$ and $\mathrm{Ubc} 9$ within this extended stretch can, however, be observed. In Ubc9 Leu81, Val86, Ser89 and Leu97 have low order parameters and for both Val86 and Leu97 a substantial contribution of 


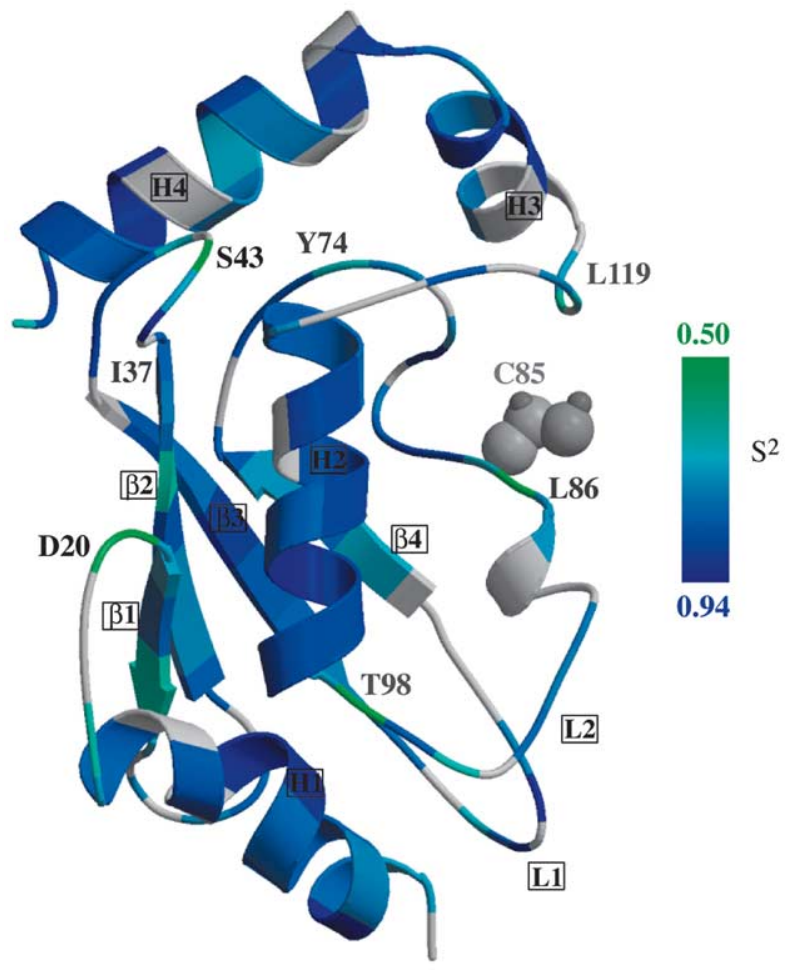

Figure 5. Dynamics in $\mathrm{UbcH} 5 \mathrm{~B}$. The ribbon representation of UbcH5B is shaded according to the $S^{2}$ values derived from the ${ }^{15} \mathrm{~N}$ relaxation analysis. The colorcoding is from blue for high $S^{2}$ values to green for low $S^{2}$ values. Residues with low order parameters are labeled. Residues for which no $S^{2}$ was determined are light gray. The active site cysteine 85 is displayed in a space-filling representation. The $\alpha$-helices (H1 to $\mathrm{H} 4$ ), $\beta$-strands ( $\beta 1$ to $\beta 4$ ) and the two loops (L1 and L2) involved in E2 binding are labeled. This Figure was generated with the programs Molscript ${ }^{66}$ and Raster3D. ${ }^{67}$

conformational exchange $R_{\mathrm{ex}}$ to the ${ }^{15} \mathrm{~N} R_{2}$ relaxation rate was detected. The corresponding residues in UbcH5B (Ile73, Ile78, Asn81 and Leu89) do not have low order parameters. The residues that do have lower order parameters in $\mathrm{UbcH} 5 \mathrm{~B}$ in this region are Tyr74 and Leu86. Although $\mathrm{UbcH} 5 \mathrm{~B}$ and Ubc9 are homologous proteins, their biological function is quite distinct. Whereas $\mathrm{UbcH} 5 \mathrm{~B}$ is primarily involved in ubiquitination of proteins, Ubc9 plays an important role in sumoylation. ${ }^{44}$ Since the differences in flexibility are mainly on the face of the protein where the active site Cys85 is located, this could reflect this different biological function.

Mutagenesis data previously shown that a highly conserved asparagine is important for the efficient catalytic activity of E2s in transferring ubiquitin to the substrate. ${ }^{24}$ In all existing X-ray structures the side-chain of this asparagine residue is hydrogen bonded to the backbone of the loop connecting the helices $\mathrm{H} 2$ and $\mathrm{H} 3$, and is thus pointing away from the active site cysteine. ${ }^{11,24}$ A structural rearrangement should therefore occur upon ubiquitination of

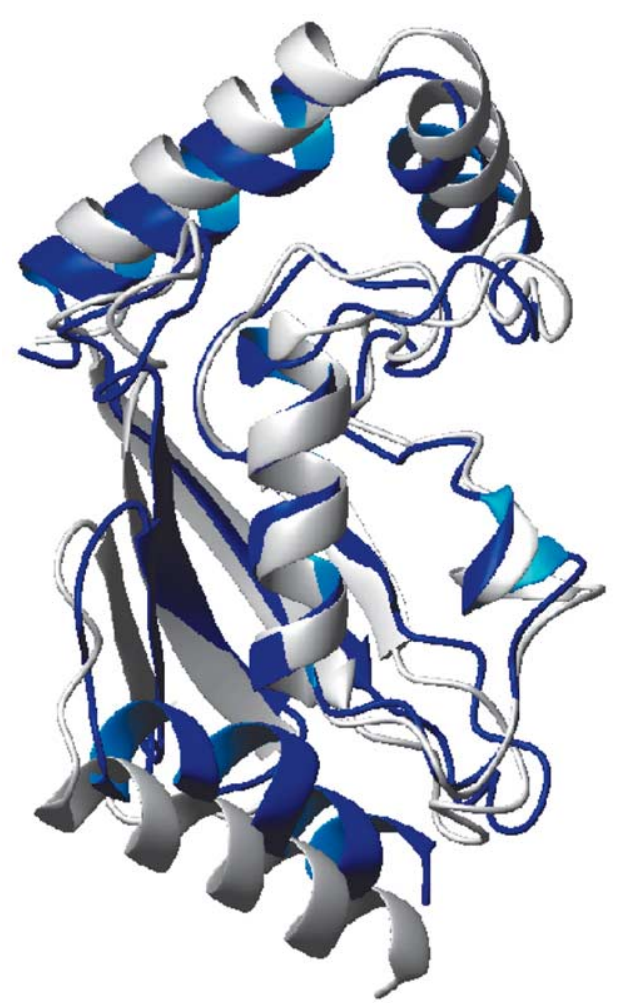

Figure 6. Overlay of the $\mathrm{UbcH} 5 \mathrm{~B}$ and yeast $\mathrm{Ubc4}$ structures (PDB: 1QCQ). The Figure shows the differential positions of helix $\mathrm{H} 1, \mathrm{H} 3$ and $\mathrm{H} 4$ in the two structures. $\mathrm{UbcH} 5 \mathrm{~B}$ is displayed blue and $\mathrm{Ubc} 4$ in white. This Figure was generated with the program MOLMOL. ${ }^{65}$

the substrate. We therefore analyzed in detail the structural and spectroscopic properties of this amino acid. The $\left({ }^{15} \mathrm{~N}-{ }^{1} \mathrm{H}\right)-\mathrm{HSQC}$ spectrum shows that the side-chain $\mathrm{NH}_{2}$ group of Asn77 has chemical shift values that are distinct from the average random coil values. The signal intensities are, however, very weak, as compared to those of the other $\mathrm{NH}_{2}$ groups, indicating the presence of conformational exchange for the Asn77 side-chain. Therefore a unique conformation for this side-chain as seen in the various X-ray structures seems unlikely in solution. In the ensemble of solution structures of $\mathrm{UbcH} 5 \mathrm{~B}$, the side-chain of Asn77 displays different conformations; in some structures it is hydrogen bonded to the backbone atoms of Asn114 as was observed in crystal structures, whereas in other structures it is either hydrogen bonded to the backbone of Asp117, to the backbone of Cys85, or solvent-exposed and in the active site cavity (Figure 7). A similar difference in orientation of the corresponding asparagine residue (Asn80) was observed only for $\mathrm{UbcH} 2 \mathrm{~B}$, for which the structure has been solved in solution as well. ${ }^{19}$ The absence of a specific and well-defined hydrogen bond between the Asn77 side-chain and the backbone of the loop between $\mathrm{H} 2$ and $\mathrm{H} 3$ is also supported by the presence of flexibility in the latter loop. Indeed Asp117, Leu119 and Val120 have lower 

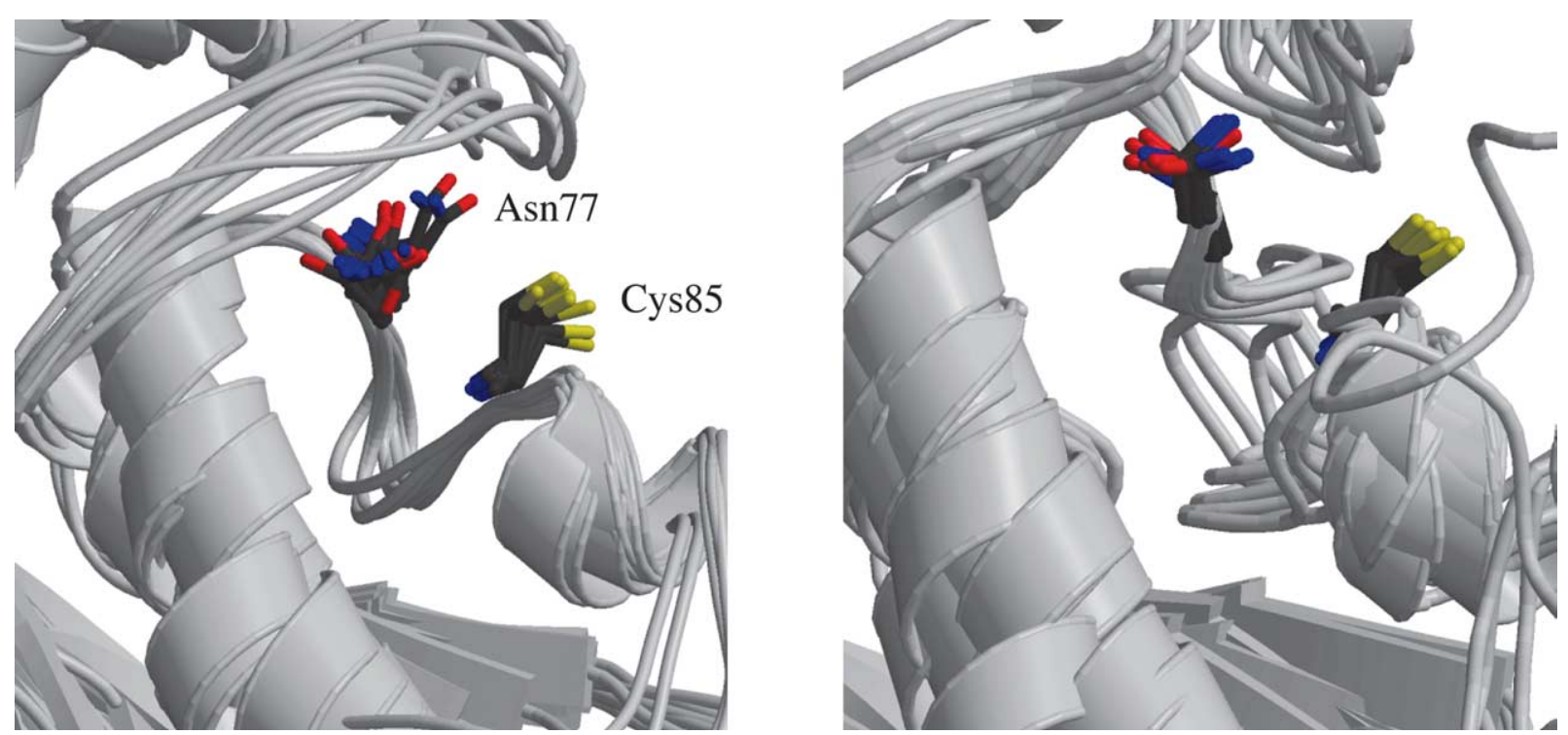

Figure 7. Comparison of the position of the asparagine residue important for oxyanion stabilization in E2-catalyzed ubiquitin conjugation. Left: NMR ensemble of UbcH5B structures (ten structures). Right: Overlay of eight E2 X-ray structures (PDB-codes: 1QCQ, 1A3S, 1AYZ, 1FZY, 1U9A, 2AAK, 2UCZ, 2EZC). This Figure was generated with the programs Molscript ${ }^{66}$ and Raster3D. ${ }^{67}$

order parameters than average (the two other residues in this loop are proline residues). In addition, Glu122 at the start of helix H3, for which no reliable relaxation parameters could be obtained, shows a low intensity peak in the HSQC spectrum, which could be an indication for the presence of conformational exchange. The same loop was also shown to be flexible in the NMR relaxation studies of Ubc9. ${ }^{43}$

Other differences between the various Ubc structures are observed in the position of the first $\alpha$-helix and the last two $\alpha$-helices. Here we find a relatively high backbone RMSD of $2.36 \AA$ between $\mathrm{UbcH} 5 \mathrm{~B}$ and Ubc4. The difference in position of these helices seems to be a general feature of the Ubc family, since most structural differences between all E2 enzymes are observed for these three helices. The precise position of the first N-terminal helix is particularly interesting, since it is part of the interacting site with ubiquitin ligases (both HECT and RING domains). ${ }^{20,21,32}$ In the UbcH5B structure many NOEs are present between the residues of this helix and residues of loops L1 (residues 56-65) and L2 (residues 89-98), which are also part of the E3 interacting site. In the structure of $\mathrm{UbcH} 5 \mathrm{~B}$, the first helix is in much closer proximity to loops L1 and L2 than in the yeast Ubc4 structure. The differential positioning of this helix with respect to the loops L1 and L2 could be one of the factors that contribute to the recognition of a different set of E3 ligases. The loop connecting the first helix (H1) and the $\beta$-sheet is flexible in both $\mathrm{UbcH} 5 \mathrm{~B}$ and Ubc9. This may allow for structural rearrangements of helix $\mathrm{H} 1$ relative to the rather rigid L1 and L2 loops to accommodate binding to different E3s. Using NMR chemical shift perturbation experiments, we already reported that residues in helix H1 (Ala2, Leu3, Arg5, Ile6, Glu9, Leu10 and Asp12) and loop L2 (Thr98, Ile99) are affected by the binding to CNOT4. ${ }^{32}$ These residues are, however, not solvent-exposed but responsible for the H1-L2 interaction, and hence chemical shift changes of these residues are probably due to a displacement of helix H1. It is therefore likely that, during binding, E2 enzymes undergo structural rearrangements involving this first helix to accommodate the different E3 ligases.

\section{Materials and Methods}

\section{Recombinant protein expression and purification}

Construction of the plasmids for expression of human UbcH5B and overexpression of ${ }^{15} \mathrm{~N}$ and ${ }^{13} \mathrm{C} /{ }^{15} \mathrm{~N}$ isotopically labeled UbcH5B have been described. ${ }^{32,45}$ The $\mathrm{UbcH5B}$ samples were concentrated to a final concentration of approximately $0.5 \mathrm{mM}$ in an NMR buffer $(150 \mathrm{mM} \mathrm{KCl}, 20 \mathrm{mM} \mathrm{KPi}(\mathrm{pH} 7.0), 10 \mu \mathrm{M} \mathrm{ZnCl}, 5 \%$ $\left.{ }^{2} \mathrm{H}_{2} \mathrm{O}\right)$.

\section{Homology modeling}

The homology model of UbcH5B was based on the structure of yeast Ubc4 (pdb: 1QCQ) ${ }^{10}$ and was generated using Modeller $4 .{ }^{46}$ For details see our previous study on the interaction between $\mathrm{UbcH} 5 \mathrm{~B}$ and the RING domain of CNOT4. ${ }^{32}$ This model was used here as a starting point for automated NOE assignment and structure calculations of UbcH5B in ARIA1.2. ${ }^{47}$

\section{NMR measurements}

For the backbone assignment of UbcH5B, 2D $\left({ }^{15} \mathrm{~N}-{ }^{1} \mathrm{H}\right)-\mathrm{HSQC}, 2 \mathrm{D}\left({ }^{13} \mathrm{C}-{ }^{1} \mathrm{H}\right)-\mathrm{HSQC}, 3 \mathrm{D} \mathrm{HNCO}, 3 \mathrm{D}$ 
$\mathrm{HNCACB}$, and 3D $\mathrm{CBCA}(\mathrm{CO}) \mathrm{NH}$ were recorded on a Bruker AVANCE $700 \mathrm{MHz}$ spectrometer. The side-chain assignment was performed using 3D TOCSY- $\left({ }^{15} \mathrm{~N}-{ }^{1} \mathrm{H}\right)-$ HSQC, 3D H(C)CH-TOCSY and 3D (H)CCH-TOCSY spectra also recorded on a Bruker AVANCE $700 \mathrm{MHz}$ spectrometer (for a review, see Sattler et al..$^{29}$ ).

The NOE spectra were recorded on Bruker AVANCE $750 \mathrm{MHz}$ and $900 \mathrm{MHz}$ spectrometers. The NOE information was extracted from two 3D NOESY- $\left({ }^{15} \mathrm{~N}-{ }^{1} \mathrm{H}\right)$ HSQC and two 2D NOE spectra (in $\mathrm{H}_{2} \mathrm{O}$ and in ${ }^{2} \mathrm{H}_{2} \mathrm{O}$ ) with mixing times of $100 \mathrm{~ms}$. The 2D NOE spectra were recorded with 768 complex points in the direct dimension (spectral width of $15151 \mathrm{~Hz}$ ) and 384 complex points in the indirect dimension (spectral width of $12,820 \mathrm{~Hz}$ ). The 3D NOESY- $\left({ }^{15} \mathrm{~N}-{ }^{1} \mathrm{H}\right)$-HSQC spectra were recorded with 768 complex points in the direct dimension (spectral width of $15,151 \mathrm{~Hz}), 160$ complex points in the ${ }^{1} \mathrm{H}$ indirect dimension (spectral width of $15,151 \mathrm{~Hz}$ ), and 40 complex points in the ${ }^{15} \mathrm{~N}$ dimension (spectral width of $3333 \mathrm{~Hz}$ ). For Fourier transformation all dimensions were zerofilled twice.

Hydrogen bond predictions were assessed from proton-deuterium exchange. A UbcH5B sample in $\mathrm{H}_{2} \mathrm{O}$ was exchanged with buffer in ${ }^{2} \mathrm{H}_{2} \mathrm{O}$. Series of $\left({ }^{15} \mathrm{~N}-{ }^{1} \mathrm{H}\right)$-HSQC were recorded as a function of time after the exchange of solvent. Amide protons, for which signals remained visible 20 hours after the exchange, were considered to be involved in a hydrogen bond. Hydrogen bond restraints were defined as two ambiguous distance restraints: one between the amide proton and any oxygen in the protein within a distance of $2.1( \pm 0.3) \AA$ and one between the amide nitrogen and any oxygen of the protein within a distance between $2.3 \AA$ and $3.3 \AA$.

All relaxation experiments were performed at $300 \mathrm{~K}$ on a Bruker AVANCE $500 \mathrm{MHz}$ spectrometer $\left({ }^{1} \mathrm{H}\right.$ frequency of $500.28 \mathrm{MHz}$ ) equipped with a QXI probe with $z$-gradients using a ${ }^{15} \mathrm{~N}$-labeled UbcH5B sample with a concentration of approximately $0.5 \mathrm{mM} .{ }^{15} \mathrm{~N} T_{1}$ and heteronuclear $\left\{{ }^{1} \mathrm{H}\right\}$-NOE values were determined using the experiments described by Farrow et al. ${ }^{48} T_{1}$ times were extracted from eight spectra with different values for the relaxation delay: $100(2 \times), 200,300,400(2 \times), 500,600$, 800 and $1000 \mathrm{~ms}$, giving $180^{\circ}$ pulses on protons every $5 \mathrm{~ms}$ to suppress cross-correlated relaxation. The heteronuclear NOE was recorded in an interleaved fashion, recording alternately one increment for the reference and one for the NOE spectrum. In the NOE experiment the protons were saturated using $120^{\circ}$ pulses $(20.7 \mathrm{kHz}) .{ }^{15} \mathrm{~N}$ $\mathrm{T}_{2}$ relaxation times were extracted from both CPMG ${ }^{49,50}$ and $T_{1 \rho}{ }^{51}$ experiments. CPMG experiments were recorded using nine different values for the relaxation delay: $0(2 \times), 16.1(2 \times), 32.2,48.2,64.3(2 \times), 80.4,96.5$, 128.6 and $160.8 \mathrm{~ms}$. During the relaxation delay ${ }^{15} \mathrm{~N} 180^{\circ}$ pulses with a field strength of $7.8 \mathrm{kHz}$ were applied every $0.95 \mathrm{~ms}\left(v_{\mathrm{CPMG}}=1 \mathrm{kHz}\right)$ and ${ }^{1} \mathrm{H} 180^{\circ}$ pulses were applied every $7.7 \mathrm{~ms}$, to suppress cross-correlated relaxation pathways. ${ }^{52}$ The $T_{1 \rho}$ experiments were recorded with varying lengths of the spin-lock pulse: $2,4,6,8,10(2 \times)$, $20,30,50(2 \times), 70,100,150 \mathrm{~ms}$. An adiabatic spin-lock pulse, as described by Mulder et al., ${ }^{53}$ was used to align the magnetization of the individual amides along their effective field. The pulse was applied on-resonance with a field-strength of $2.5 \mathrm{kHz}$. The number of ${ }^{1} \mathrm{H} 180^{\circ}$ pulses during the relaxation period was adapted to the used relaxation delay. ${ }^{54}$ No ${ }^{1} \mathrm{H} 180^{\circ}$ pulses were applied up to $30 \mathrm{~ms}$, one ${ }^{1} \mathrm{H} 180^{\circ}$ pulse was applied in the middle of the $50 \mathrm{~ms}$ and $70 \mathrm{~ms}$ delays, two pulses in the case of $100 \mathrm{~ms}$ and three for the $150 \mathrm{~ms}$ delay.

All spectra were processed with NMRPipe ${ }^{55}$ and analyzed using NMRView5.0.4. ${ }^{56}$ Relaxation parameters were extracted and analyzed with the program Curvefit $^{57}, \dagger$ using a two-parameter fitting and a Monte Carlo simulation to estimate the errors.

\section{Diffusion anisotropy}

In the absence of large amplitude internal motions and conformational exchange, the ratio of the ${ }^{15} \mathrm{~N}$ transverse and longitudinal relaxation rates $\left(R_{2} / R_{1}\right)$ is dependent on the angles $\theta$ and $\phi$ between the amide bond vector and the diffusion tensor of the protein. Given a good estimate of the diffusion tensor components $\left(D_{x x}, D_{y y}\right.$ and $\left.D_{z z}\right)$, the $R_{2} / R_{1}$ ratios can thus be used to refine the orientation of the amide bond vectors. ${ }^{25}$ These ratios are included in $\mathrm{CNS}^{58}$ as diffusion anisotropy restraints (DANI) as described by Tjandra et al. ${ }^{25}$ The diffusion tensor components are defined by the overall rotational correlation time $\left(\tau_{c}\right)$, the anisotropy $(A)$ and the rhombicity $(\eta)$ :

$$
\begin{gathered}
\tau_{c}=\frac{1}{2 \cdot\left(D_{x x}+D_{y y}+D_{z z}\right)} \\
A=\frac{2 D_{z z}}{\left(D_{y y}+D_{x x}\right)} \\
\eta=\frac{1.5 \cdot\left(D_{y y}-D_{x x}\right)}{D_{z z}-0.5 \cdot\left(D_{y y}+D_{x x}\right)}
\end{gathered}
$$

In $\mathrm{CNS}^{58}$ the geometric content of the $R_{2} / R_{1}$ ratios is incorporated in the simulated annealing protocol for the structure calculation by minimizing the harmonic potential energy term $E_{\text {dani: }}$ :

$$
E_{\text {dani }}=k_{\text {dani }} \sum\left\{\left[\left(R_{2} / R_{1}\right)_{\text {calc }}-\left(R_{2} / R_{1}\right)_{\text {obs }}\right]^{2}\right\}
$$

where $k_{\text {dani }}$ is the force constant for the diffusion anisotropy restraints and $\left(R_{2} / R_{1}\right)_{\text {calc }}$ and $\left(R_{2} / R_{1}\right)_{\text {obs }}$ are the back-calculated and observed $R_{2} / R_{1}$ ratios, respectively.

The selection of $R_{2} / R_{1}$ ratios to determine the diffusion tensor components and to define diffusion anisotropy restraints is as follows. All residues are selected that have both a heteronuclear NOE value higher than 0.65 and an $R_{2}$ rate smaller than the average rate plus one standard deviation. In addition, residues with a high $R_{2}$ rate and a corresponding $R_{1}$ rate lower than the average rate minus one standard deviation are also selected. This selection procedure keeps the residues with high $R_{2}$ values caused by the anisotropy of the system. ${ }^{59}$ In this way, $87 R_{2} / R_{1}$ ratios were selected. The diffusion tensor components were determined in three different ways. (i) From the average of the highest, the lowest and the most frequently occurring $R_{2} / R_{1}$ ratios in the distribution (Figure 2(a)) the tensor components can be estimated as has been described by Clore et al. ${ }^{60}$ This gave a value of 1.61 for the anisotropy $A$ and 0.33 for the rhombicity $\eta$. (ii) Since an average of the extreme values is used in the first approach, the anisotropy is rather under-than overestimated. Therefore a more extreme estimate, using the averages of the two minimum and maximum values minus and plus the standard deviation, respectively, gives a value of 1.68 for $A$ with a corresponding $\eta$ of 0.27 . (iii) When a proper structural model is available, TENSOR2 $^{39}$ can be used to determine the rotational diffusion tensor, based on the selected set of relaxation

$\dagger$ http:/ /cpmcnet.columbia.edu/dept/gsas/biochem/ labs/palmer/software/curvefit.html 
rates. Using the homology model of UbcH5B the computed values are 1.64 for $A$ and 0.31 for $\eta$, respectively. These three sets of values for the diffusion tensor were used to refine the homology model of UbcH5B (see below).

The rotational diffusion parameters of a molecule can also be estimated by hydrodynamic modeling. This has been implemented in the program HYDRONMR, ${ }^{40}$ which uses a bead shell method to perform the hydrodynamic calculations. An important parameter in these calculations is the radius $a$ of the spherical elements that are used to replace each non-hydrogen atom to build the initial shell model. For most proteins this atomic element radius $a$ has a value between $2.8 \AA$ and $3.8 \AA$, with a distribution centered at $3.3 \AA{ }^{41}$ To calculate the hydrodynamic properties of UbcH5B we used a value of $3.2 \AA$ for $a$ and a solvent viscosity of $0.8 \mathrm{cP}$ at a temperature of $300 \mathrm{~K}$.

The agreement between back-calculated and experimental $R_{2} / R_{1}$ ratios was evaluated by the reduced $\chi^{2}$ :

$$
\chi^{2}=\frac{1}{N} \sum\left\{\frac{\left[\left(R_{2} / R_{1}\right)_{\mathrm{obs}}-\left(R_{2} / R_{1}\right)_{\mathrm{calc}}\right]^{2}}{\sigma_{R_{2} / R_{1}}^{2}}\right\}
$$

where $\sigma$ is the error in the ratios and $N$ the number of observables.

\section{Structure calculation}

The automated assignment and structure calculations of UbcH5B were performed with ARIA1.2 ${ }^{33}$ using CNS. ${ }^{58}$ The topallhdg5.3.pro ${ }^{61}$ topology and parameter set was used based on the PROLSQ parameters. ${ }^{62}$ The ARIA1.2 scripts were modified to allow the use of diffusion anisotropy restraints as defined in CNS. ${ }^{25} \mathrm{~A}$ grid search procedure to optimize the initial values for the anisotropic $(A)$ and rhombic $(\eta)$ components of the diffusion tensor, based on the calculated structures, was introduced. Herein the sum of the restraint energy term $E_{\text {dani }}$ over the ensemble of structures is minimized by a grid search of \pm 0.1 and \pm 0.05 around the starting values of the diffusion tensor components $A$ and $\eta$, in steps of 0.02 and 0.01 , respectively.

First, the homology model of $\mathrm{UbcH} 5 \mathrm{~B}$ was refined using TALOS dihedral and diffusion anisotropy restraints in explicit water. This refinement was performed starting from three different sets of estimated values of the rotational diffusion tensor (see above), resulting in three ensembles of each 20 structures. The force constants for the diffusion anisotropy and TALOS dihedral angle restraints were set to $10 \mathrm{kcal} \mathrm{mol}^{-1}$ and $200 \mathrm{kcal} \mathrm{mol}^{-1} \mathrm{rad}^{-2}$, respectively. Based on the resulting 60 models the diffusion tensor components were optimized using the grid search described above and the resulting values were used in the subsequent structure calculation runs. The automated NOE assignment was performed in three steps as described below.

(i) First the 60 models were used to create an initial set of NOE assignments, without calculating any structure. Because of the sensitivity of the $900 \mathrm{MHz}$ spectrometer and the size of the protein, the upper bound limit for the NOE calibration was set to $7 \AA$ and spin diffusion correction ${ }^{63}$ was used in all runs.

(ii) In the second run, consisting of nine ARIA iterations, the assigned peaks from the first run were used to calculate an initial ensemble of structures, including diffusion anisotropy and TALOS dihedral restraints. After each iteration the ambiguous NOEs were reassigned and recalibrated based on the 20 lowest energy structures and rejected if violated in more than $50 \%$ of the structures. The ambiguity cut-off was reduced from 1.01 in the first iteration to a final value of 0.90 in the last iteration. The violation tolerance was set to $1000 \AA$ for the first two iterations and then progressively reduced to $0.1 \AA$ in the last iteration. In the last iteration, the tensor components were optimized using a grid search as described above. For the structure calculations a simulated annealing (SA) protocol consisting of four stages was used starting from an extended conformation using both torsion angle dynamics (TAD) and Cartesian dynamics. (i) The high temperature TAD stage consisted of 10,000 steps at $10,000 \mathrm{~K}$. This was followed by (ii) an 8000 steps TAD cooling stage with a final temperature of $2000 \mathrm{~K}$, (iii) a 5000 steps first Cartesian cooling stage to $1000 \mathrm{~K}$, and (iv) a 10,000 steps second Cartesian cooling stage to $50 \mathrm{~K}$. During the SA protocol the force constants for the TAD stages, first Cartesian cooling stage and second Cartesian cooling stage were set to 10,50 and $50 \mathrm{kcal} \mathrm{mol}^{-1} \AA^{-2}$ for the NOE restraints, to 50 , 100 and $200 \mathrm{kcal} \mathrm{mol}^{-1} \mathrm{rad}^{-2}$ for the dihedral restraints and to 1,1 and $10 \mathrm{kcal} \mathrm{mol}^{-1}$ for the diffusion anisotropy restraints, respectively. The number of calculated structures in the iterations was 100 (50) for iterations 1 and 2, 20 (20) for iterations 3 to 7,50 (20) for iteration 8 and 100 (50) for the final iteration, with the structures that were kept in the subsequent iteration indicated between brackets.

(iii) In the third and last run the final ensemble of structures was calculated, starting in the first iteration from the ensemble of ten lowest energy structures of run 2. The NOE assignments of run 2 were used and kept fixed. In addition the peak lists containing the remaining unassigned NOEs were introduced, to allow the assignment of more NOEs. The ambiguity cut-off was 0.90 in all iterations and the violation tolerance was reduced from 1.0 to $0.1 \AA$ during the nine iterations, with only an increased value of 1.0 for iteration 6 . In this run the hydrogen bond restraints were added with the same force as the NOE. The SA protocol described above was used for the structure calculations. The number of calculated structures was 50 (25) for iteration 2 to 8 and 100 (50) for the last iteration, with the structures that were kept in the subsequent iteration between brackets. After each iteration an optimization of the diffusion tensor components was performed, based on the ensemble of 20 lowest energy structures. The 50 final lowest energy structures were refined in explicit water using the OPLS parameters. ${ }^{64}$

\section{Data Bank accession numbers}

Chemical shifts of UbcH5B have been deposited to the BioMagResBank with entry number 6277. The coordinates of the ten best structures of UbcH5B have been deposited in the RCSB Protein Data Bank with accession number $1 \mathrm{~W} 4 \mathrm{U}$.

\section{Acknowledgements}

This investigation was supported by the Research 
Council for the Chemical Sciences of the Netherlands Organization for Scientific Research (NWO-CW). This work was also supported by NWO "Jonge Chemici" and "Pionier" grants to A. M. J. J. B., and H. Th. M. T., respectively. C. D. was supported financially by the Center for Biomedical Genetics. The authors are grateful to Aart Nederveen for helpful discussion.

\section{References}

1. Weissman, A. M. (2001). Themes and variations on ubiquitylation. Nature Rev. Mol. Cell. Biol. 2, 169-178.

2. Pickart, C. M. (2001). Mechanisms underlying ubiquitination. Annu. Rev. Biochem. 70, 503-533.

3. Glickman, M. H. \& Ciechanover, A. (2002). The ubiquitin-proteasome proteolytic pathway: destruction for the sake of construction. Physiol. Rev. 82, 373-428.

4. Seufert, W. \& Jentsch, S. (1990). Ubiquitin-conjugating enzymes UBC4 and UBC5 mediate selective degradation of short-lived and abnormal proteins. EMBO J. 9, 543-550.

5. Scheffner, M., Huibregtse, J. M. \& Howley, P. M. (1994). Identification of a human ubiquitin-conjugating enzyme that mediates the E6-AP-dependent ubiquitination of p53. Proc. Natl Acad. Sci. USA, 91, 8797-8801.

6. Gonen, H., Bercovich, B., Orian, A., Carrano, A., Takizawa, C., Yamanaka, K. et al. (1999). Identification of the ubiquitin carrier proteins, E2s, involved in signal-induced conjugation and subsequent degradation of IкB $\alpha$. J. Biol. Chem. 274, 14823-14830.

7. Stancovski, I., Gonen, H., Orian, A., Schwartz, A. L. \& Ciechanover, A. (1995). Degradation of the protooncogene product c-Fos by the ubiquitin proteolytic system in vivo and in vitro: identification and characterization of the conjugating enzymes. Mol. Cell. Biol. 15, 7106-7116.

8. Aguilar, R. C. \& Wendland, B. (2003). Ubiquitin: not just for proteasomes anymore. Curr. Opin. Cell. Biol. 15, 184-190.

9. Cook, W. J., Jeffrey, L. C., Sullivan, M. L. \& Vierstra, R. D. (1992). Three-dimensional structure of a ubiquitin-conjugating enzyme (E2). J. Biol. Chem. 267, 15116-15121.

10. Cook, W. J., Jeffrey, L. C., Xu, Y. \& Chau, V. (1993). Tertiary structures of class I ubiquitin-conjugating enzymes are highly conserved: crystal structure of yeast Ubc4. Biochemistry, 32, 13809-13817.

11. Tong, H., Hateboer, G., Perrakis, A., Bernards, R. \& Sixma, T. K. (1997). Crystal structure of murine/ human Ubc9 provides insight into the variability of the ubiquitin-conjugating system. J. Biol. Chem. 272, 21381-21387.

12. Cook, W. J., Martin, P. D., Edwards, B. F. P., Yamazaki, R. K. \& Chau, V. (1997). Crystal structure of a class I ubiquitin conjugating enzyme (Ubc7) from Saccharomyces cerevisiae at 2.9 angstroms resolution. Biochemistry, 36, 1621-1627.

13. Worthylake, D. K., Prakash, S., Prakash, L. \& Hill, C. P. (1998). Crystal structure of the Saccharomyces cerevisiae ubiquitin-conjugating enzyme Rad6 at $2.6 \AA$ resolution. J. Biol. Chem. 273, 6271-6276.

14. Jiang, F. \& Basavappa, R. (1999). Crystal structure of the cyclin-specific ubiquitin-conjugating enzyme from clam, E2-C, at $2.0 \AA$ resolution. Biochemistry, 38, 6471-6478.

15. Hamilton, K. S., Ellison, M. J., Barber, K. R., Williams, R. S., Huzil, J. T., McKenna, S. et al. (2001). Structure of a conjugating enzyme-ubiquitin thiolester intermediate reveals a novel role for the ubiquitin tail. Structure, 9, 897-904.

16. VanDemark, A. P., Hofmann, R. M., Tsui, C., Pickart, C. M. \& Wolberger, C. (2001). Molecular insights into polyubiquitin chain assembly: crystal structure of the Mms2/Ubc13 heterodimer. Cell, 105, 711-720.

17. Lin, Y., Hwang, W. C. \& Basavappa, R. (2002). Structural and functional analysis of the human mitotic-specific ubiquitin-conjugating enzyme, UbcH10. J. Biol. Chem. 277, 21913-21921.

18. Giraud, M. F., Desterro, J. M. P. \& Naismith, J. H. (1998). Structure of ubiquitin-conjugating enzyme 9 displays significant differences with other ubiquitinconjugating enzymes which may reflect its specificity for sumo rather than ubiquitin. Acta Crystallog. sect. D, 54, 891-898.

19. Miura, T., Klaus, W., Ross, A., Güntert, P. \& Senn, H. (2002). The NMR structure of the class I human ubiquitin-conjugating enzyme 2b. J. Biomol. NMR, 22, 89-92.

20. Huang, L., Kinnucan, E., Wang, G., Beaudenon, S., Howley, P. M., Huibregtse, J. M. \& Pavletich, N. P. (1999). Structure of an E6AP-UbcH7 complex: insights into ubiquitination by the E2-E3 enzyme cascade. Science, 286, 1321-1326.

21. Zheng, N., Wang, P., Jeffrey, P. D. \& Pavletich, N. P. (2000). Structure of a c-Cbl-UbcH7 complex: RING domain function in ubiquitin-protein ligases. Cell, 102, 533-539.

22. Moraes, T. F., Edwards, R. A., McKenna, S., Pastushok, L., Xiao, W., Glover, J. N. \& Ellison, M. J. (2001). Crystal structure of the human ubiquitin conjugating enzyme complex, hMms2-hUbc13. Nature Struct. Biol. 8, 669-673.

23. Bernier-Villamor, V., Sampson, D. A., Matunis, M. J. \& Lima, C. D. (2002). Structural basis for E2-mediated SUMO conjugation revealed by a complex between ubiquitin-conjugating enzyme Ubc9 and RanGAP1. Cell, 108, 345-356.

24. Wu, P. Y., Hanlon, M., Eddins, M., Tsui, C., Rogers, R. S., Jensen, J. P. et al. (2003). A conserved catalytic residue in the ubiquitin-conjugating enzyme family. EMBO J. 22, 5241-5250.

25. Tjandra, N., Garrett, D. S., Gronenborn, A. M., Bax, A. \& Clore, G. M. (1997). Defining long range order in NMR structure determination from the dependence of heteronuclear relaxation times on rotational diffusion anisotropy. Nature Struct. Biol. 4, 443-449.

26. Tjandra, N., Feller, S. E., Pastor, R. W. \& Bax, A. (1995). Rotational diffusion anisotropy of human ubiquitin from ${ }^{15} \mathrm{~N}$ NMR relaxation. J. Am. Chem. Soc. 117, 12562-12566.

27. Brüschweiler, R., Liao, X. B. \& Wright, P. E. (1995). Long-range motional restrictions in a multidomain zinc-finger protein from anisotropic tumbling. Science, 268, 886-889.

28. Farrow, N. A., Archer, S. J., Wu, Z. J., Camac, D. M., Parsons, T., Rolfe, M. \& Domaille, P. J. (2000). Backbone resonance assignment of human UBC4. J. Biomol. NMR, 18, 363-364.

29. Sattler, M., Schleucher, J. \& Griesinger, C. (1999). Heteronuclear multidimensional NMR experiments 
for the structure determination of proteins in solution employing pulsed field gradients. Prog. Nucl. Magn. Reson. Spectrosc. 34, 93-158.

30. Cornilescu, G., Delaglio, F. \& Bax, A. (1999). Protein backbone angle restraints from searching a database for chemical shift and sequence homology. J. Biomol. NMR, 13, 289-302.

31. Loria, J. P., Rance, M. \& Palmer, A. G. (1999). A relaxation-compensated Carr-Purcell-Meiboom-Gill sequence for characterizing chemical exchange by NMR spectroscopy. J. Am. Chem. Soc. 121, 2331-2332.

32. Dominguez, C., Bonvin, A. M. J. J., Winkler, G. S., van Schaik, F. M. A., Timmers, H. T. M. \& Boelens, R. (2004). Structural model of the UbcH5B/CNOT4 complex revealed by combining NMR, mutagenesis and docking approaches. Structure, 12, 633-644.

33. Linge, J. P., O'Donoghue, S. I. \& Nilges, M. (2001). Automated assignment of ambiguous nuclear Overhauser effects with ARIA. Methods Enzymol. 339, 71-90.

34. Güntert, P. (2003). Automated NMR protein structure calculation. Prog. Nucl. Magn. Reson. Spectrosc. 43, 105-125.

35. Herrmann, T., Güntert, P. \& Wüthrich, K. (2002). Protein NMR structure determination with automated NOE assignment using the new software CANDID and the torsion angle dynamics algorithm DYANA. J. Mol. Biol. 319, 209-227.

36. Duggan, B. M., Legge, G. B., Dyson, H. J. \& Wright, P. E. (2001). SANE (structure assisted NOE evaluation): an automated model-based approach for NOE assignment. J. Biomol. NMR, 19, 321-329.

37. Hare, B. J. \& Wagner, G. (1999). Application of automated NOE assignment to three-dimensional structure refinement of a $28 \mathrm{kDa}$ single-chain $\mathrm{T}$ cell receptor. J. Biomol. NMR, 15, 103-113.

38. Güntert, P., Berndt, K. D. \& Wüthrich, K. (1993). The program ASNO for computer-supported collection of NOE upper distance constraints as input for proteinstructure determination. J. Biomol. NMR, 3, 601-606.

39. Dosset, P., Hus, J. C., Blackledge, M. \& Marion, D. (2000). Efficient analysis of macromolecular rotational diffusion from heteronuclear relaxation data. J. Biomol. NMR, 16, 23-28.

40. Garcia de la Torre, J., Huertas, M. L. \& Carrasco, B. (2000). HYDRONMR: prediction of NMR relaxation of globular proteins from atomic-level structures and hydrodynamic calculations. J. Magn. Reson. 147, 138-146.

41. Bernado, P., Garcia de la Torre, J. \& Pons, M. (2002). Interpretation of ${ }^{15} \mathrm{~N}$ NMR relaxation data of globular proteins using hydrodynamic calculations with HYDRONMR. J. Biomol. NMR, 23, 139-150.

42. Lipari, G. \& Szabo, A. (1982). Model-free approach to the interpretation of nuclear magnetic resonance relaxation in macromolecules. 2. Analysis of the experimental results. J. Am. Chem. Soc. 104, 4559-4570.

43. Liu, Q., Yuan, Y. C., Shen, B. H., Chen, D. J. \& Chen, Y. (1999). Conformational flexibility of a ubiquitin conjugation enzyme (E2). Biochemistry, 38, 1415-1425.

44. Muller, S., Hoege, C., Pyrowolakis, G. \& Jentsch, S. (2001). SUMO, ubiquitin's mysterious cousin. Nature Rev. Mol. Cell. Biol. 2, 202-210.

45. Albert, T. K., Hanzawa, H., Legtenberg, Y. I. A., de Ruwe, M. J., van den Heuvel, F. A. J., Collart, M. A. et al. (2002). Identification of a ubiquitin-protein ligase subunit within the CCR4-NOT transcription repressor complex. EMBO J. 21, 355-364.
46. Sali, A. \& Blundell, T. L. (1993). Comparative protein modelling by satisfaction of spatial restraints. J. Mol. Biol. 234, 779-815.

47. Nilges, M., Macias, M. J., O'Donoghue, S. I. \& Oschkinat, H. (1997). Automated NOESY interpretation with ambiguous distance restraints: the refined NMR solution structure of the pleckstrin homology domain from $\beta$-spectrin. J. Mol. Biol. 269, 408-422.

48. Farrow, N. A., Muhandiram, D. R., Singer, A. U., Pascal, S. M., Kay, C. M., Gish, G. et al. (1994). Backbone dynamics of a free and a phosphopeptidecomplexed Src homology 2 domain studied by ${ }^{15} \mathrm{~N}$ NMR relaxation. Biochemistry, 33, 5984-6003.

49. Meiboom, S. \& Gill, D. (1958). Modified spin-echo method for measuring nuclear relaxation times. Rev. Sci. Instrum. 29, 688-691.

50. Carr, H. Y. \& Purcell, E. M. (1954). Effects of diffusion on free precession in nuclear magnetic resonance experiments. Phys. Rev. 94, 630-638.

51. Peng, J. W., Thanabal, V. \& Wagner, G. (1991). 2D heteronuclear NMR measurements of spin-lattice relaxation-times in the rotating frame of $X$ nuclei in heteronuclear HX spin systems. J. Magn. Reson. 94, 82-100.

52. Kay, L. E., Nicholson, L. K., Delaglio, F., Bax, A. \& Torchia, D. A. (1992). Pulse sequences for removal of the effects of cross-correlation between dipolar and chemical-shift anisotropy relaxation mechanism on the measurement of heteronuclear T1 and T2 values in proteins. J. Magn. Reson. 97, 359-375.

53. Mulder, F. A. A., de Graaf, R. A., Kaptein, R. \& Boelens, R. (1998). An off-resonance rotating frame relaxation experiment for the investigation of macromolecular dynamics using adiabatic rotations. J. Magn. Reson. 131, 351-357.

54. Korzhnev, D. M., Skrynnikov, N. R., Millet, O., Torchia, D. A. \& Kay, L. E. (2002). An NMR experiment for the accurate measurement of heteronuclear spin-lock relaxation rates. J. Am. Chem. Soc. 124, 10743-10753.

55. Delaglio, F., Grzesiek, S., Vuister, G. W., Zhu, G., Pfeifer, J. \& Bax, A. (1995). NMRPipe: a multidimensional spectral processing system based on UNIX pipes. J. Biomol. NMR, 6, 277-293.

56. Johnson, B. A. \& Blevins, R. A. (1994). NMRView: a computer program for the visualization and analysis of NMR data. J. Biomol. NMR, 4, 603-614.

57. Palmer, A. G., Rance, M. \& Wright, P. E. (1991). Intramolecular motions of a zinc finger DNA-binding domain from Xfin characterized by proton-detected natural abundance ${ }^{13} \mathrm{C}$ heteronuclear NMRspectroscopy. J. Am. Chem. Soc. 113, 4371-4380.

58. Brunger, A. T., Adams, P. D., Clore, G. M., DeLano, W. L., Gros, P., Grosse-Kunstleve, R. W. et al. (1998). Crystallography and NMR system: a new software suite for macromolecular structure determination. Acta Crystallog. sect. D, 54, 905-921.

59. Pawley, N. H., Wang, C. Y., Koide, S. \& Nicholson, L. K. (2001). An improved method for distinguishing between anisotropic tumbling and chemical exchange in analysis of ${ }^{15} \mathrm{~N}$ relaxation parameters. J. Biomol. NMR, 20, 149-165.

60. Clore, G. M., Gronenborn, A. M., Szabo, A. \& Tjandra, N. (1998). Determining the magnitude of the fully asymmetric diffusion tensor from heteronuclear relaxation data in the absence of structural information. J. Am. Chem. Soc. 120, 4889-4890.

61. Linge, J. P., Williams, M. A., Spronk, C. A. E. M., 
Bonvin, A. M. J. J. \& Nilges, M. (2003). Refinement of protein structures in explicit solvent. Proteins: Struct. Funct. Genet. 50, 496-506.

62. Engh, R. A. \& Huber, R. (1991). Accurate bond and angle parameters for $\mathrm{X}$-ray protein-structure refinement. Acta Crystallog. sect. A, 47, 392-400.

63. Linge, J. P., Habeck, M., Rieping, W. \& Nilges, M. (2004). Correction of spin diffusion during iterative automated NOE assignment. J. Magn. Reson. 167, 334-342.

64. Jorgensen, W. L. \& Tirado-Rives, J. (1988). The OPLS potential functions for proteins. Energy minimizations for crystals of cyclin peptides and crambin. J. Am. Chem. Soc. 110, 1657-1666.

65. Koradi, R., Billeter, M. \& Wüthrich, K. (1996). MOLMOL: a program for display and analysis of macromolecular structures. J. Mol. Graph. 14, 51-55 (see also pages 29-32).

66. Kraulis, P. J. (1991). MOLSCRIPT: a program to produce both detailed and schematic plots of protein structures. J. Appl. Crystal. 24, 946-950.

67. Merritt, E. A. \& Murphy, M. E. P. (1994). Raster3D version 2.0: a program for photorealistic molecular graphics. Acta Crystallog. sect. D, 50, 869-873.

Edited by M. F. Summers

(Received 30 July 2004; received in revised form 17 September 2004; accepted 20 September 2004) 